\title{
Therapeutic anti-EGFR antibody 806 generates responses in murine de novo EGFR mutant-dependent lung carcinomas
}

\author{
Danan Li, ${ }^{1,2}$ Hongbin Ji, ${ }^{1,2}$ Sara Zaghlul,,1,2 Kate McNamara, ${ }^{1,2}$ Mei-Chih Liang, ${ }^{1,2}$ \\ Takeshi Shimamura, ${ }^{1}$ Shigeto Kubo, ${ }^{3}$ Masaya Takahashi, ${ }^{3}$ Lucian R. Chirieac, ${ }^{4}$ \\ Robert F. Padera, ${ }^{4}$ Andrew M. Scott, ${ }^{5}$ Achim A. Jungbluth, ${ }^{6}$ Webster K. Cavenee, ${ }^{7}$ \\ Lloyd J. Old, ${ }^{6}$ George D. Demetri, ${ }^{1,8}$ and Kwok-Kin Wong ${ }^{1,8}$
}

1Department of Medical Oncology, Dana-Farber Cancer Institute, Boston, Massachusetts, USA. 2Ludwig Center at Dana-Farber/Harvard Cancer Center, Boston, Massachusetts, USA. ${ }^{3}$ Department of Radiology, Beth Israel Deaconess Medical Center, Boston, Massachusetts, USA. ${ }^{4}$ Department of Pathology, Brigham and Women's Hospital, Boston, Massachusetts, USA. 'Ludwig Institute for Cancer Research, Melbourne Tumor Biology Branch, Melbourne, Victoria, Australia. ${ }^{6}$ Ludwig Institute for Cancer Research, New York, New York, USA. ${ }^{7}$ Ludwig Institute for Cancer Research, San Diego Branch, Center for Molecular Genetics, Department of Medicine, and Cancer Center, University of California, San Diego, San Diego, California, USA. ${ }^{8}$ Department of Medicine, Brigham and Women's Hospital and Harvard Medical School, Boston, Massachusetts, USA.

\begin{abstract}
Activating EGFR mutations occur in human non-small cell lung cancer (NSCLC), with $5 \%$ of human lung squamous cell carcinomas having EGFRvIII mutations and approximately $10 \%-30 \%$ of lung adenocarcinomas having EGFR kinase domain mutations. An EGFR-targeting monoclonal antibody, mAb806, recognizes a conformational epitope of WT EGFR as well as the truncated EGFRvIII mutant. To explore the anticancer spectrum of this antibody for EGFR targeted cancer therapy, $\mathrm{mAb806}$ was used to treat genetically engineered mice with lung tumors that were driven by either EGFRvIII or EGFR kinase domain mutations. Our results demonstrate that $\mathrm{mAb806}$ is remarkably effective in blocking EGFRvIII signaling and inducing tumor cell apoptosis, resulting in dramatic tumor regression in the EGFRvIII-driven murine lung cancers. Another EGFR-targeting antibody, cetuximab, failed to show activity in these lung tumors. Furthermore, treatment of murine lung tumors driven by the EGFR kinase domain mutation with $\mathrm{mAb806}$ also induced significant tumor regression, albeit to a less degree than that observed in EGFRvIII-driven tumors. Taken together, these data support the hypothesis that mAb806 may lead to significant advancements in the treatment of the population of NSCLC patients with these 2 classes of EGFR mutations.
\end{abstract}

\section{Introduction}

Targeted cancer therapy is designed to disrupt the function of specific molecules needed for carcinogenesis and tumor growth and thus either kills or prevents the growth of cancer cells (1). In contrast to conventional cytotoxic chemotherapy, such targeted cancer therapy may be more effective and less harmful to normal cells. A major effort in the targeted cancer therapy field has been the development of agents that target the EGFR. EGFR is a member of the ErbB family of closely related receptors including EGFR (ErbB-1), Her2/neu (ErbB-2), Her3 (ErbB-3), and Her4 (ErbB-4). Activation of EGFR leads to receptor tyrosine kinase activation and a series of downstream signaling events that mediate cellular proliferation, motility, adhesion, invasion, and resistance to chemotherapy as well as inhibition of apoptosis (2-4), processes that are crucial to the continual proliferation and survival of cancer cells.

To date, 2 major types of anti-EGFR agents have entered the clinical setting: anti-EGFR antibodies and small-molecule EGFR

Nonstandard abbreviations used: NSCLC, non-small cell lung cancer; TKI, tyrosine kinase inhibitor.

Conflict of interest: A.A. Jungbluth, W.K. Cavenee, L.J. Old, and A.M. Scott assigned their $\mathrm{mAb806}$ patent rights to the Ludwig Institute for Cancer Research, which has licensed the 806 antibody to Life Science Pharmaceuticals (LSP). L.J. Old serves as a member of the board of directors for LSP. G.D. Demetri and A.M. Scott have a consulting relationship with LSP.

Citation for this article: J. Clin. Invest. 117:346-352 (2007). doi:10.1172/JCI30446. tyrosine kinase inhibitors (TKIs) $(5,6)$. Anti-EGFR antibodies such as cetuximab were designed to bind to the extracellular domain of the EGFR and block activation of EGFR downstream signaling (7). In contrast, small-molecule TKIs such as gefitinib or erlotinib compete with ATP for binding to the intracellular catalytic domain of the EGFR tyrosine kinase and thus prevent EGFR autophosphorylation and downstream signaling (4).

Both of these anti-EGFR drug groups have shown some clinical efficacy in a subset of patients with a wide variety of cancers. Treatment with gefitinib or erlotinib of patients with lung cancer having EGFR kinase domain mutations often generates dramatic clinical responses $(5,8)$. However, the effectiveness of gefitinib or erlotinib in lung adenocarcinoma with WT EGFR or in other histological subtypes, such as squamous cell carcinoma, is limited (9, 10). Furthermore, it has been shown in preclinical and clinical trials that gefitinib or erlotinib are largely ineffective in inhibiting the function of the EGFRvIII mutant (11), a distinct activating EGFR mutation in which there is an in-frame deletion of exons II-VII. EGFRvIII is commonly found in glioblastomas and recently found to be present in a subset of human lung squamous cell carcinomas (12) and a large fraction of head and neck cancers (13). Cetuximab was shown to be effective in a small subset of non-small cell lung cancer (NSCLC) patients and patients with head and neck cancers, as well as colorectal cancer patients. However, the response to cetuximab does not seem to correlate with expression levels of EGFR. Thus, it is unclear why these patients respond while other 

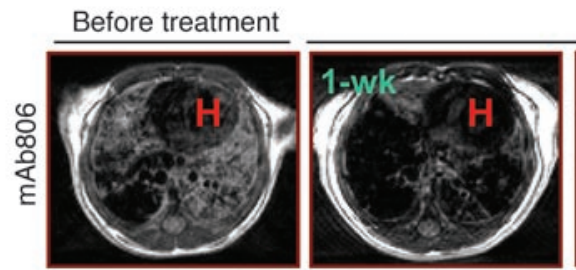

After treatment
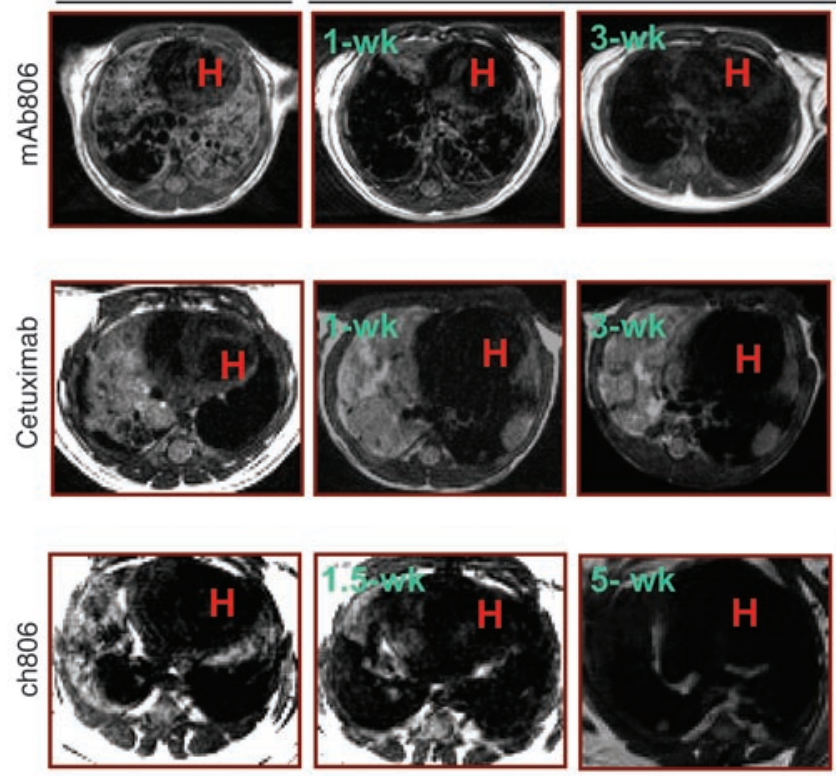
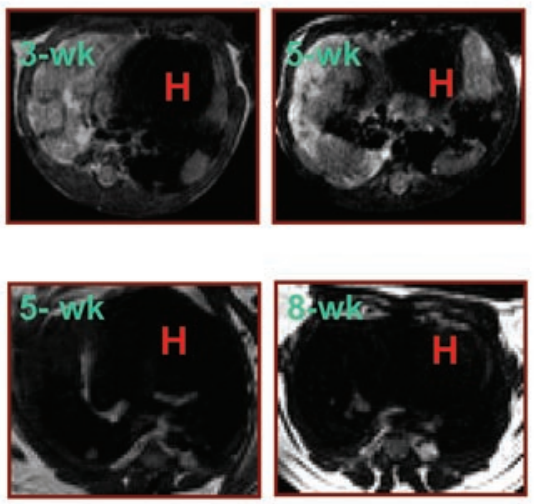
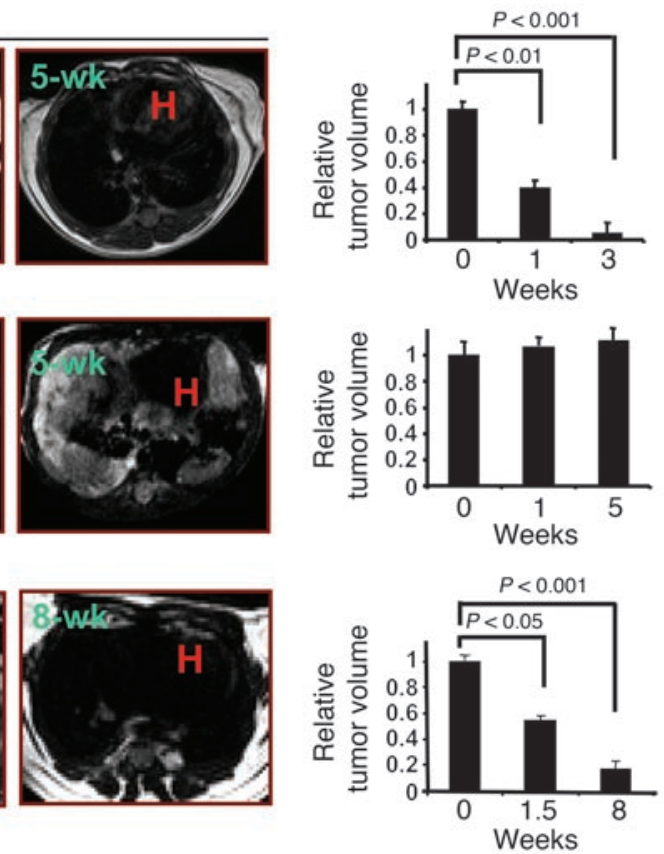

Figure 1

Murine lung tumors driven by EGFRvIll expression are sensitive to mAb806 and ch806 antibody treatment but resistant to cetuximab treatment. Tet-op-EGFRvIII/CCSP-rtTA, Ink4A/Arf-- mice were treated with either mAb806 or ch806 at $0.5 \mathrm{mg}$ per dose or cetuximab at $1 \mathrm{mg}$ per dose through daily i.p. injection. Antibodies were given every 2 days at the same dose after the first week of treatment. Serial MRI was performed at the indicated time points, and corresponding sections of representative mice in each treatment group are shown. Data (expressed as mean \pm SD) illustrate the tumor regression measured by MRI, and statistical analyses were performed using 2-tailed unpaired Student's $t$ test. All mice were kept on a doxycycline diet throughout the experiment. $\mathrm{H}$ indicates the area of the heart.

cancer patients whose tumors have high EGFR expression are refractory to cetuximab treatment (14).

$\mathrm{mAb} 806$ is a novel murine antibody, originally raised to recognize the unique truncation mutant, EGFRvIII (15-17). Importantly, the epitope recognized by mAb806 is not accessible in inactive WT EGFR but is exposed in a transitional form of WT EGFR in cells with overexpression of EGFR and expression of EGFRvIII (18). The epitope studies are supported by immunohistochemical studies demonstrating that the 806 antibody binds to epitopes present in gliomas, as well as a broad range of epithelial cancers, but not to normal human tissues $(16,19)$. These and other preclinical data suggest that mAb806 might have a different spectrum of clinical activity and side-effect profile distinct from those of cetuximab and other anti-EGFR antibodies. In xenograft models, mAb806 exhibited a potent antitumor activity with no targeting of normal tissues. Thus, the unique targeting capabilities of mAb806 represent what we believe to be a new paradigm for cancer-specific molecularly targeted therapy.

Recent studies have shown that $10 \%-30 \%$ of NSCLC patients have EGFR kinase domain mutations, while $5 \%$ of lung squamous cell carcinoma patients have the extracellular domain EGFRvIII mutation $(12,20)$. To investigate the clinical potential of mAb806 in cancer-specific targeted therapy in NSCLC patients harboring EGFR mutations, we utilized 2 established mouse lung cancer models that are dependent on EGFRvIII or EGFR kinase domain mutants. Our data show that $\mathrm{mAb} 806$ is very effective in the treatment of murine NSCLC driven by expression of either EGFRvIII or EGFR kinase domain mutation and suggest that this antibody is likely to have clinical activity in patients whose tumors have similar mutations.

\section{Results}

Treatment with mAb806, but not cetuximab, induces tumor regression in mice bearing lung tumors with EGFRvIII mutation. Our previous studies have established the essential role of EGFRvIII mutation in tumor maintenance of murine lung tumors driven by the mutation. Blocking of EGFRvIII activation resulted in dramatic tumor regression associated with apoptosis in the de novo murine lung cancer model (12). Tet-op-EGFR $v I I I / C C S P-r t T A$, Ink4A/Arf-/- mice developed lung adenocarcinomas with features of bronchoalvealor carcinoma after approximately 8-10 weeks of doxycycline administration (Figure 1, far-left panels, and Figure 2A, top row). After tumor-bearing mice were identified by MRI, a daily i.p. injection of a 0.5 -mg dose of mAb806 was given for the first week and then every 2 days for the following 4 weeks. Serial MRI was performed at the end of 1,3 , and 5 weeks of treatment to determine changes in tumor volume and/or density. Tumor reduction was notable by MRI after 1 week of mAb806 treatment (average reduction of $60 \% \pm 5 \%$ among 6 mice; Figure 1, top row). Tumor burden continued to decrease after 3 weeks of treatment (average reduction of $95 \% \pm 8 \%$ ), and all 6 mice had complete tumor regression after 5 weeks of treatment. In contrast, treatment of mice with cetuximab was unable to induce tumor regression in 4 Tet-op-EGFR III/ CCSP-rtTA, Ink $4 \mathrm{~A} / \mathrm{Arf}^{-/-}$mice even after 5 weeks of treatment at $1 \mathrm{mg} /$ mouse with the same dosing schedule. We also observed that the mice treated with cetuximab became progressively frailer and that some even died because of significant tumor burden during the treatment period (data not shown).

Pathologic examination of lungs from these mice correlated with the MRI findings: a decrease in tumor cellularity was present 
A

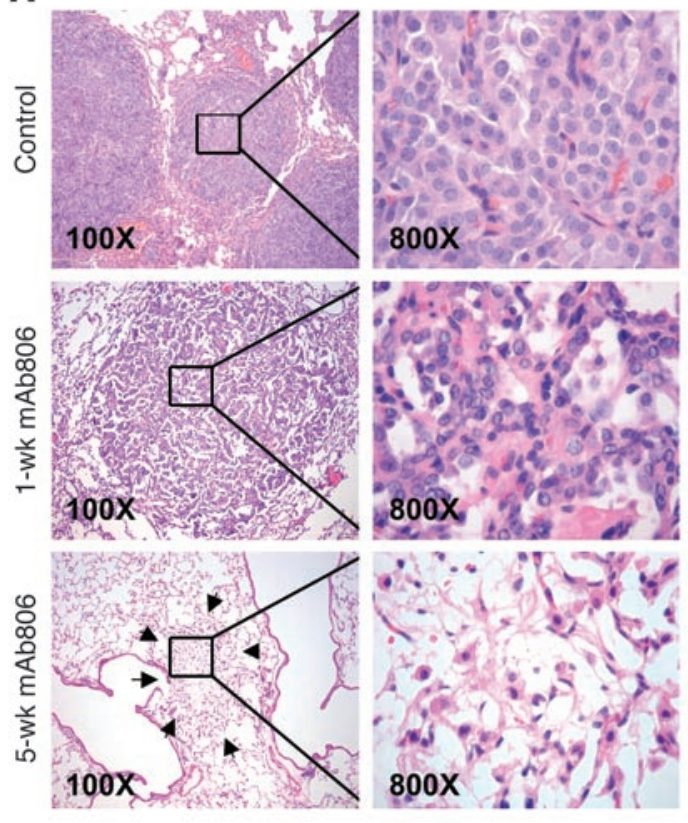

B
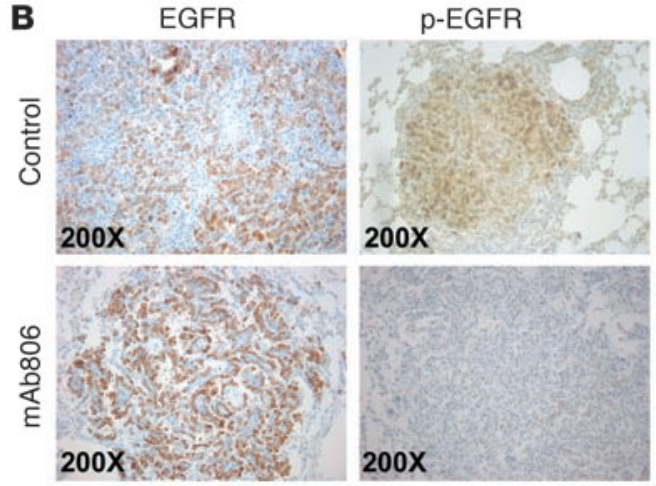

C
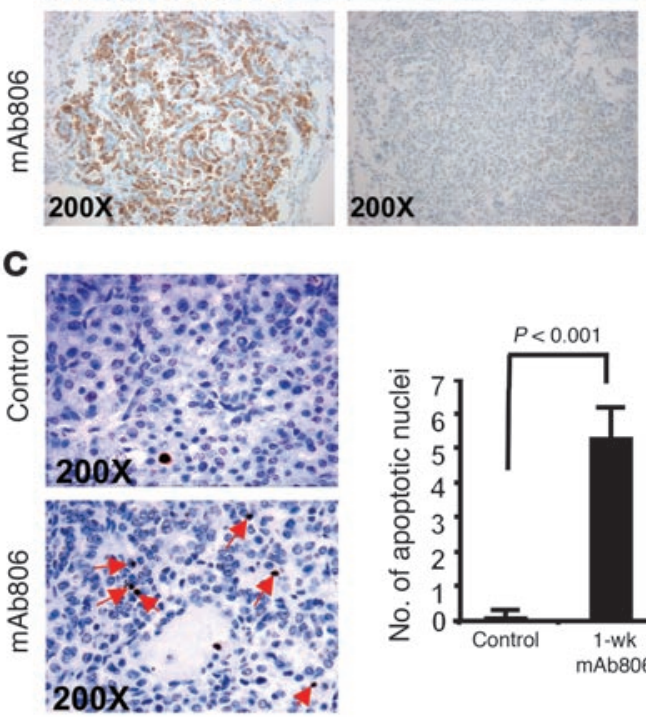

$200 \mathrm{x}$

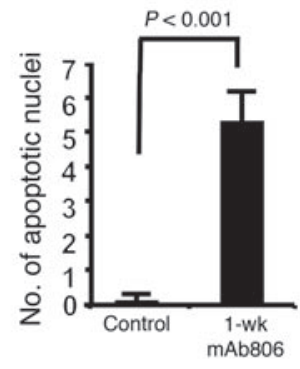

Figure 2

Histopathological features of EGFRvIll-driven lung adenocarcinomas in mice treated with mAb806. (A) Lung adenocarcinoma driven by EGFRvIII expression for more than 8 weeks (top row). After 1 week of treatment with mAb806, tumors became smaller and had increased fibrosis (middle row). Lung specimens were grossly normal when mAb806 treatment ended at 5 weeks (bottom row). Arrows show a fibrotic nodule, consisting of fibroblasts and macrophages. No tumor cells were found in this particular fibrosis area. Original magnification, $\times 100$ (left column), $\times 800$ (right column). (B) Similar patterns and intensities of immunohistological staining of total EGFR can be observed in control mice and mice treated with mAb806 for 1 week (top- and bottom-left panels, respectively); intensity of phospho-EGFR staining of tumor cells decreased after 1 week of treatment (bottom-right panel) compared with that in untreated tumors (top-right panel). Representative photos were taken under $\times 200 \mathrm{magnification}$. (C) TUNEL staining shows an increase in the number of apoptotic nuclei (red arrows) in EGFRvlll-driven lung tumors after 1 week of treatment with mAb806 (bottom-left panel) compared with untreated tumors (top-left panel). Representative photos were taken under $\times 200$ magnification. Data (expressed as mean \pm SD) represent the apoptotic indices in lung tumors before and after 1 week of mAb806 treatment, determined from at least 200 high-power fields (HPF). Statistical analyses were performed using 2-tailed unpaired Student's $t$ test (right panel).

in adenocarcinomas after 1 week of treatment with mAb806 (Figure $2 \mathrm{~A}$, middle row). After 5 weeks, lungs had focal fibrosis and scarring, with sparse monocytic cell infiltrates, potentially representing areas of continuing remodeling from regressed tumors (Figure 2A, bottom row). Although live cancer cells could still be rarely observed in several foci of these fibrotic nodules, most of the fibrosis and scarring area did not contain any tumor cells. In contrast, the tumors from mice treated with cetuximab appeared to be unaffected, showing no visible histological difference from untreated tumors (data not shown). Thus, treatment with mAb806 antibody led to rapid and dramatic tumor regression in the EGFR $v I I$-driven mouse lung cancer model, while cetuximab treatment was largely ineffective.

$m A b 806$ inhibits EGFRvIII phosphorylation and induces apoptosis of tumor cells in Tet-op-EGFRvIII/CCSP-rtTA, Ink4A/Arf-/- mice. To determine whether the mAb806 that was administered i.p. recognized its target in the lung tumors, we performed immunohistochemical staining in lung tumors of mice treated with or without mAb806 using antibodies against total EGFR and phospho-EGFR. As expected, mAb806 treatment had no impact on the total EGFRvIII expression in tumor cells (Figure 2B). However, the expression of
phospho-EGFRvIII diminished after 1 week of mAb806 treatment (Figure 2B). We next confirmed these findings by immunoblot analysis using lung lysates collected at different time points during treatment with mAb806. The level of phospho-EGFRvIII decreased dramatically after 1 week of mAb806 treatment, while the total EGFRvIII level remained similar to that of untreated controls (Figure 3), indicating a strong inhibitory effect of mAb806 on EGFRvIII phosphorylation. Interestingly, the total EGFRvIII level did finally decrease after 5 weeks of mAb806 administration. One explanation for this could be the dramatic decrease in the number of viable tumor cells. Consistent with this interpretation, greatly increased TUNEL staining was observed in lung tumors after 1 week of mAb806 treatment compared with untreated tumors (Figure $2 \mathrm{C}$ ). Along with its effects on phospho-EGFR levels, 1 week of mAb806 treatment also decreased phospho-Akt and phosphoErk1/2 expression; these EGFR downstream signaling molecules are functionally associated with antiapoptosis and proliferation pathways. Surprisingly, we observed a weak but reproducible increase in the phospho-Akt level after 5 weeks of mAb806 treatment when compared with 1 week of treatment. It is unlikely that this phosphorylation of Akt was initiated by EGFRvIII, as 


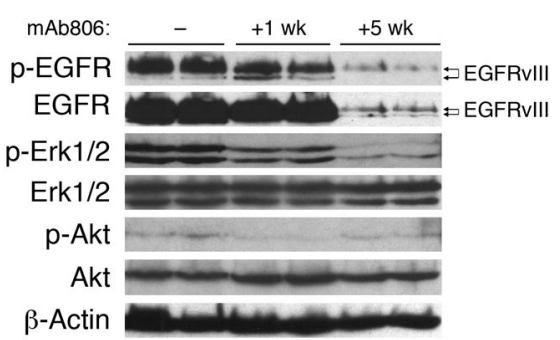

phospho-EGFRvIII expression was low at this time point. Possibly, Akt could have been activated by other signaling events that were involved in lung remodeling process. These data suggest that mAb806 induces tumor regression in EGFRvIII mice by blocking EGFR activation and increasing tumor cell apoptosis.

ch806 treatment leads to dramatic tumor regression in murine lung tumors with EGFRvIII mutation. ch806 is a humanized form of mAb806 (21). To determine whether the humanized antibody could be as efficient as the murine mAb806 in the treatment of lung adenocarcinoma in vivo, we treated tumor-bearing Tet-op-EGFRvIII/CCSP-rtTA, Ink $4 \mathrm{~A} / \mathrm{Arf}^{-1-}$ mice with 1 dose of $0.5 \mathrm{mg}$ of ch806 by i.p. injection daily for the first week and then 1 dose every 2 days for another 7 weeks. These mice underwent reimaging at $1.5,5$, and 8 weeks of treatment and were then sacrificed for histological analysis. We observed a dramatic reduction in tumor volume by MRI scanning starting from 1.5 weeks of the treatment ( $43 \% \pm 3 \%)$, and near complete tumor regression $(83 \% \pm 7 \%)$ was achieved at 8 weeks of treatment in each of the 4 mice being treated with ch806 (Figure 1, bottom panel). The histology of mice treated with ch806 (data not shown) was similar to that of mice after mAb806 treatment and was consistent with the MRI data.

ch806 is effective in treating murine lung tumors with EGFR L858R mutation. To address whether ch806 could be effective against

\section{Figure 3}

Western blot analysis of whole lung lysates from mAb806-treated Tetop-EGFRvIII/CCSP-rtTA, Ink4A/Arf-1- mice. Whole lung lysates from mice at different time points of $\mathrm{mAb806}$ treatment were analyzed. Inhibition of EGFR phosphorylation was observed after 1 week of treatment, while total EGFR levels decreased after 5 weeks of treatment. Erk1/2 phosphorylation was inhibited throughout the mAb806 administration; Akt phosphorylation decreased at 1 week of treatment and slightly increased after 5 weeks of treatment when compared with the 1-week time point. $\beta$-Actin served as a loading control.

EGFR kinase domain mutation-driven lung cancer, EGFR L858RIRES-Luciferase/CCSP-rtTA mice were employed. ch806 was administered at $0.5 \mathrm{mg} / \mathrm{mouse}$ every day for 4 weeks, and serial MRI scanning of all treated mice was performed at the end of 1,2 , and 4 weeks of treatment. Tumor regression was observed after 2 weeks of ch806 treatment $(21 \% \pm 2 \%)$ and was $41 \% \pm 2 \%$ at 4 weeks of ch806 treatment (Figure 4A). Histologically, the lungs of ch806treated mice showed an increased diffuse cellular infiltrate with macrophages, especially in areas surrounding the remaining viable tumors. Furthermore, macrophages were present in multiple areas of the tumors, suggesting that macrophage-mediated cytotoxicity might be one of the underlying mechanisms of antibody-induced tumor regression (Figure 4B). It should also be noted that the presence of consolidation within the lung due to increased accumulation of macrophages associated with tumor cells could overestimate tumor volume by MRI.

\section{Discussion}

EGFR mutations and activation events are common in human malignancies, including NSCLC. Activation of EGFR signaling can occur through receptor overexpression as well as by constitutive signaling due to gain-of-function mutant forms of EGFR. Approximately $10 \%-30 \%$ of NSCLC patients have EGFR
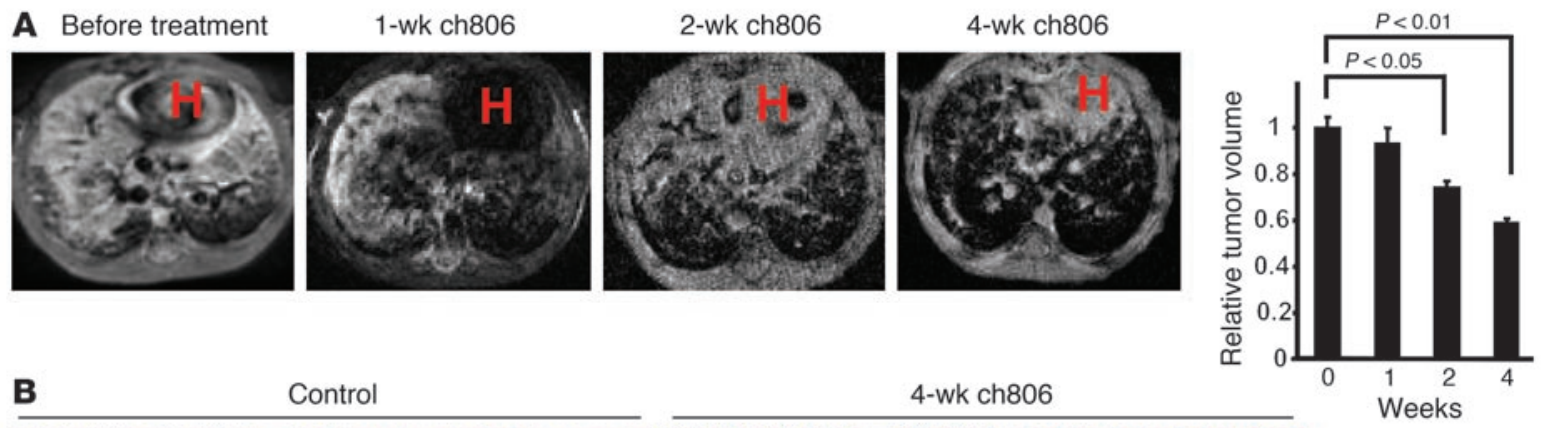

B

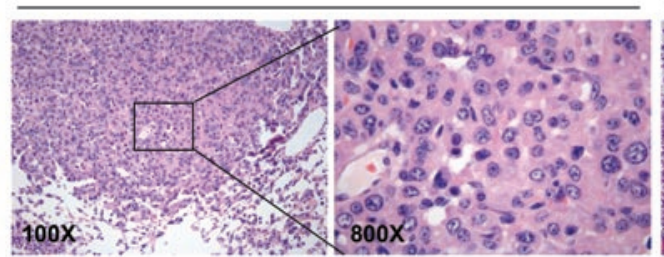

4-wk ch806

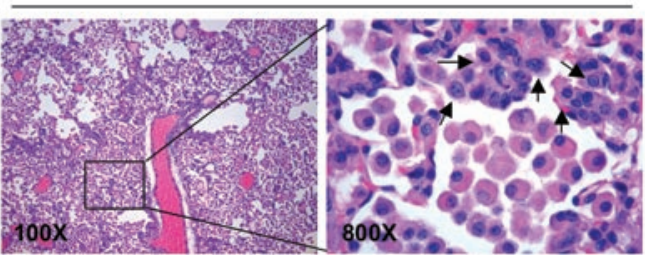

\section{Figure 4}

EGFR kinase domain mutation L858R-driven mouse lung adenocarcinoma responds to ch806 treatment. (A) Tet-op-EGFR L858R-IRES-Luciferase/CCSP-rtTA mice were treated with ch806 at $0.5 \mathrm{mg}$ per dose by daily i.p. injection for 4 weeks. MRI showed decreased tumor volume after 2 and 4 weeks of treatment. Data (expressed as mean \pm SD) illustrate the tumor regression measured by MRI, and statistical analyses were performed using 2-tailed unpaired Student's $t$ test. (B) Histopathological analysis shows shrinkage of tumors and marked macrophage infiltration in ch806-treated Tet-op-EGFR L858R-IRES-Luciferase/CCSP-rtTA mice (right 2 panels), when compared with untreated Tet-op-EGFR L858RIRES-Luciferase/CCSP-rtTA control mice (left 2 panels). Arrows show foci of residual tumors. 
kinase domain mutations in their lung tumors, and about $5 \%$ of patients with squamous cell lung cancer have the specific EGFR$v I I I$ extracellular domain mutation $(12,20)$. Here we show that mAb806 and its humanized form, ch806, are effective in treating murine lung cancers with both types of EGFR mutations. The dramatic tumor regression observed was associated with blockage of EGFRvIII signaling and, consequently, increased apoptosis. The response to ch806 was not as impressive as that reported for erlotinib and cetuximab in mice with lung tumors having EGFR kinase domain mutations, although they did have an objective response $(41 \% \pm 2 \%)$ radiographically and histologically $(22,23)$. In contrast, nearly complete tumor regression was achieved in mice with EGFRvIII-driven lung tumors after treatment with mAb806, while cetuximab was without effect. This latter result is perhaps not surprising, since cetuximab is designed to interfere with the interaction between ligand and the EGFR extracellular domain (24). It has been established that the EGFRvIII mutation leads to conformational changes and exhibits constitutive kinase activity independent of ligand stimulation, which contributes to the tumor formation (25). Although cetuximab has been approved by the FDA for cancer patients, there is no clear biomarker to predict the efficacy of treatment with this antibody in individual patients, since response rates and overall survival are not correlated with EGFR protein expression by immunohistochemistry (14).

Although small-molecule TKIs are effective in the treatment of many NSCLC patients with EGFR kinase domain mutations, all patients eventually develop resistance associated with a secondary mutation, T790M $(10,26)$. Consistently, in vitro studies have shown that tumor cells with T790M mutations are resistant to treatment with erlotinib $(27,28)$. Evidence from the crystal structure of the EGFR kinase domain with a secondary T790M mutation indicates that there should be little effect of T790M mutation on the receptor function. It may be that the T790M mutation interferes with erlotinib for its binding to the ATPase pocket (27). Nonetheless, the extracellular domain of the T790M mutant potentially provides a good target for antibody-based cancer therapy including cetuximab and mAb806. This could mean that NSCLC tumors with secondary T790M point mutations, which are resistant to small TKI treatment, might respond to mAb806 treatment. Efforts to generate mice harboring the compound mutant EGFR alleles containing both the activating kinase domain mutations and the T790M mutation are ongoing in order to test this hypothesis.

Recently released data from a phase I clinical trial have shown that the ch806 antibody, unlike cetuximab, selectively binds to tumor cells of lung cancers, including squamous cell lung carcinoma, but not to normal tissues (29). No significant toxicities of the ch806 antibody were observed in this trial. In comparison with other EGFR targeted cancer therapies, including cetuximab and TKI treatments, ch806 appears to have a much greater specificity, targeting a conformationally dependent epitope of the EGFR on cancer cells while sparing WT EGFR on most, if not all, normal cells. Our results clearly indicate the effectiveness of mAb 806 on blocking EGFR signaling. Thus, the unique targeting capabilities of ch806 represent what we believe to be a new and exciting paradigm for cancer-specific molecularly targeted therapy, which may benefit patients whose cancers are dependent upon uncontrolled EGFR signaling due to overexpression or to gain-of-function mutations including EGFRvIII or EGFR kinase domain mutations.

\section{Methods}

Mouse cohorts. The generation of Tet-op-EGFRvIII/CCSP-rtTA, Ink4A/Arf ${ }^{-1-}$ mice and Tet-op-EGFR L858R-IRES-Luciferase/CCSP-rtTA mice was described previously $(12,22)$. All mice were housed in a pathogen-free environment at the Harvard School of Public Health, and all mouse experiments performed were approved by the Institutional Animal Care and Use Committee of Harvard University. Littermates were used as controls in all experiments. To induce EGFRvIII and EGFR L858R expression, mice were fed a doxycycline diet (Research Diets Inc.). Doxycycline withdrawal experiments in previous studies clearly indicated that lung tumors from both alleles are solely dependent on doxycycline.

Targeted therapies using either mAb806 or ch806 or cetuximab in vivo. Mice on continuous doxycycline diets for more than 8 weeks underwent MRI to document the lung tumor burden. mAb806 or ch806 (generated by the Ludwig Institute for Cancer Research, Melbourne) was delivered into mice bearing lung tumors through i.p. injection at daily $0.5-\mathrm{mg}$ doses. After the 1 -week treatment, antibodies were administered every 2 days at the same dose for the additional indicated weeks. Cetuximab (obtained commercially from Bristol-Myers Squibb) was administered to mice by i.p. injection at 1 $\mathrm{mg}$ per dose using the same dosing schedule. Mice were imaged with MRI at the indicated time points to determine reduction in tumor volume and then sacrificed for further histological and biochemical studies after the completion of treatment. All the mice were kept on the doxycycline diet throughout the experiments. Littermates were used as controls for all the drug treatment studies.

Pathologic assessment of lung tumors. Mice were euthanized at the indicated times, and the left lungs were dissected and snap-frozen for biochemical analysis. The right lungs were then inflated under pressure $(25 \mathrm{~cm})$ with neutral buffered $10 \%$ formalin for 10 minutes and fixed overnight. H\&E stains were performed on $5-\mu \mathrm{m}$-thick sections from formalin-fixed, paraffin-embedded tumor samples in the Department of Pathology at Brigham and Women's Hospital.

Immunohistochemical analysis was performed on formalin-fixed paraffin sections. Slides were deparaffinized in xylene and rehydrated sequentially in ethanol. For antibodies requiring antigen retrieval, antigen-unmasking solution (Vector Laboratories) was used according to the manufacturer's instructions. Slides were quenched in hydrogen peroxide $(0.3 \%-3 \%)$ to block endogenous peroxidase activity and then washed in automation buffer (Fisher Scientific). Slides were blocked in 5\% normal serum for 1 hour at room temperature and then incubated overnight at $4{ }^{\circ} \mathrm{C}$ with primary antibody diluted in blocking buffer. The avidin-biotin-peroxidase complex method (VECTASTAIN ABC kit; Vector Laboratories) was used, and slides were counterstained with hematoxylin. Slides were dehydrated sequentially in ethanol, cleared with xylene, and mounted with Permount (Fisher Scientific). Biotinylated DBA lectin (Vector Laboratories) was used at 1:100. The antibodies used were total EGFR and phospho-EGFR Y1068 (1:50; Cell Signaling Technology). Apoptosis was measured by counting positive cells using the TUNEL assay (ApopTag kit; Chemicon International).

Western blot analysis. Snap-frozen lung tissue samples were homogenized in RIPA buffer (Boston BioProducts) containing the Complete Protease Inhibitors Cocktail and Phosphatase Inhibitors Cocktail Set I and II (EMD Biosciences). Lung lysates were cleared by centrifugation and boiled in $1 \times$ final SDS sample buffer (50 mM Tris [pH 6.8], $10 \%$ glycerol, $0.715 \mathrm{M}$ $\beta$-mercaptoethanol, $2 \%$ SDS, and $0.01 \%$ bromophenol blue) for 5 minutes. Lysates were then separated by SDS-PAGE, transferred to nitrocellulose membranes, and detected by immunoblotting with antibodies using SuperSignal West Pico Chemiluminescent Substrate (Pierce Biotechnology). The antibodies used in this study were directed against total EGFR, phospho-EGFR (pY1068), total Akt, phospho-Akt (pS473), total Erk1/2 and phospho-Erk1/2 (pT202/pY204) (all from Cell Signaling Technology); 
and $\beta$-actin (Santa Cruz Biotechnology Inc.). Antibodies were used according to the conditions recommended by the manufacturers.

MRI and tumor volume measurement. Animals were anesthetized with $1.5 \%-2 \%$ isoflurane (IsoFlo; Abbott) mixed in $100 \%$ oxygen via a nose cone. In order to control for the effects of motion, both cardiac and respiratory gating was applied to all MRI studies. Since the acquisition of the magnetic resonance signal is synchronized with the cardiac and respiratory cycles, the magnetic resonance signal was acquired at each cardiac phase and at the endexpiratory phase, allowing motion artifacts to be significantly reduced.

MRI protocols optimized for assessing pulmonary parenchyma and vessels in normal mice (30) were adapted for operation at 4.7 Tesla (BioSpec 47/40; Bruker BioSpin). The system is equipped with shielded gradient systems with a maximum power gradient of $30 \mathrm{G} / \mathrm{cm}$ and a cardiac-respiratory triggering system (BioTrig; Bruker BioSpin). Then the animals were placed prone, with the electrodes (both forepads and left rear pad) for cardiac gating and a respiratory sensor on their bodies, and headfirst into the system, with the thorax centered with respect to the center of the radio frequency birdcage coil (inner diameter, $3 \mathrm{~cm}$ ). For the purpose of reproducible positioning of the imaging region, a low-resolution multislice image, serving as the end-expiratory phase localizer, was first acquired for the entire lung in both transverse and coronal planes using a fast-spin echo sequence (RARE [rapid acquisition with relaxation enhancement]; repetition time $[\mathrm{TR}] /$ effective echo time $[\mathrm{TE}]=1,000 / 28 \mathrm{~ms}$, bandwidth $=50 \mathrm{kHz}$, field of view $=30 \mathrm{~mm}$, matrix $=128 \times 128$, slice thickness $=1 \mathrm{~mm}$, number of excitations $=1$ ). Further, $2 \mathrm{D}$ multislice gradient echo imaging was performed in multislice transverse and coronal planes encompassing the entire lung with cardiac-respiratory gating. We selected a pulse TR less than the duration of 1 cardiac cycle (ranging from 150 to $200 \mathrm{~ms}$; average, $178 \mathrm{~ms}$ ), where $1 \mathrm{k}$-space line was filled for each image per single heartbeat. The minimum TE (1.8 ms) was used to reduce the susceptibility effect arising from the interface between air and tissue or between bone and tissue, which would otherwise have reduced the magnetic resonance signal. Other scan parameters were: flip angle $=22^{\circ}$, matrix size $=256 \times 256$, field of view $=2.56 \mathrm{~cm}^{2}$, slice thickness $=1 \mathrm{~mm}$, and number of excitations $=4$, affording a $100-\mu \mathrm{m}^{2}$ in-plane resolution. Total scan time was approximately 6-7 minutes in each plane, depending on the individual animal's cardiac/respiratory rates. On each magnetic resonance image, the areas indicating the pulmonary tumor were manually segmented and measured to calculate tumor volumes using NIH ImageJ (version 1.33; http://rsb.info.nih.gov/ij/).

Statistics. All the statistical analyses were performed using unpaired 2-tailed Student's $t$ test. $P$ values of less than 0.05 were considered significant.

\section{Acknowledgments}

We thank Jeffrey Whitsett for providing the CCSP-rtTA-transgenic mice; Terry Johns (Ludwig Institute for Cancer Research, Melbourne) and Frank Furnari (Ludwig Institute for Cancer Research, San Diego) for helpful discussions. We also thank Sarah White and Denise Collins for critical comments. W.K. Cavenee was supported in part by a grant from the National Cancer Institute (CA95616) and a Fellow Award from the National Foundation for Cancer Research. A.M. Scott was supported in part by grant 280912 from the National Health and Medical Research Council. K.-K. Wong was supported by NIH grants K08AG 2400401 and R01 CA122794, the Cecily and Robert Harris Foundation, Joan's Legacy, and the Sidney Kimmel Foundation for Cancer Research.

Received for publication September 24, 2006, and accepted in revised form November 28, 2006.

Address correspondence to: Kwok-Kin Wong, Department of Medical Oncology, Lowe Center for Thoracic Oncology, Dana-Farber Cancer Institute, Harvard Medical School, 44 Binney Street, D810, Boston, Massachusetts 02115, USA. Phone: (617) 632-6084; Fax: (617) 582-7839; E-mail: Kwong1@partners.org.

Danan Li and Hongbin Ji contributed equally to this work.
1. Ji, H., Sharpless, N.E., and Wong, K.K. 2006. EGFR target therapy: view from biological standpoint. Cell Cycle. 5:2072-2076.

2. Hynes, N.E., and Lane, H.A. 2005. ERBB receptors and cancer: the complexity of targeted inhibitors. Nat. Rev. Cancer. 5:341-354.

3. Arteaga, C.L. 2003. ErbB-targeted therapeutic approaches in human cancer. Exp. Cell Res. 284:122-130.

4. Mendelsohn, J., and Baselga, J. 2000. The EGF receptor family as targets for cancer therapy. Oncogene. 19:6550-6565.

5. Snyder, L.C., Astsaturov, I., and Weiner, L.M. 2005. Overview of monoclonal antibodies and small molecules targeting the epidermal growth factor receptor pathway in colorectal cancer. Clin. Colorectal Cancer. 5(Suppl. 2):S71-S80.

6. Herbst, R.S. 2002. Targeted therapy in nonsmall-cell lung cancer. Oncology (Williston Park). 16(9 Suppl. 9):19-24.

7. Groner, B., Hartmann, C., and Wels, W. 2004. Therapeutic antibodies. Curr. Mol. Med. 4:539-547.

8. Haber, D.A., et al. 2005. Molecular targeted therapy of lung cancer: EGFR mutations and response to EGFR inhibitors. Cold Spring Harb. Symp. Quant. Biol. 70:419-426.

9. Park, K., and Goto, K. 2006. A review of the benefit-risk profile of gefitinib in Asian patients with advanced non-small-cell lung cancer. Curr. Med. Res. Opin. 22:561-573.

10. Sakurada, A., Shepherd, F.A., and Tsao, M.S. 2006. Epidermal growth factor receptor tyrosine kinase inhibitors in lung cancer: impact of primary or secondary mutations. Clin. Lung Cancer.
7(Suppl. 4):S138-S144.

11. Halatsch, M.E., Schmidt, U., Behnke-Mursch, J., Unterberg, A., and Wirtz, C.R. 2006. Epidermal growth factor receptor inhibition for the treatment of glioblastoma multiforme and other malignant brain tumours. Cancer Treat. Rev. 32:74-89.

12. Ji, H., et al. 2006. Epidermal growth factor receptor variant III mutations in lung tumorigenesis and sensitivity to tyrosine kinase inhibitors. Proc. Natl. Acad. Sci. U. S. A. 103:7817-7822.

13. Sok, J.C., et al. 2006. Mutant epidermal growth factor receptor (EGFRvIII) contributes to head and neck cancer growth and resistance to EGFR targeting. Clin. Cancer Res. 12:5064-5073.

14. Italiano, A. 2006. Targeting the epidermal growth factor receptor in colorectal cancer: advances and controversies. Oncology. 70:161-167.

15. Jungbluth, A.A., et al. 2003. A monoclonal antibody recognizing human cancers with amplification/overexpression of the human epidermal growth factor receptor. Proc. Natl. Acad. Sci. U.S. A. 100:639-644.

16. Luwor, R.B., et al. 2001. Monoclonal antibody 806 inhibits the growth of tumor xenografts expressing either the de2-7 or amplified epidermal growth factor receptor (EGFR) but not wild-type EGFR. Cancer Res. 61:5355-5361.

17. Mishima, K., et al. 2001. Growth suppression of intracranial xenografted glioblastomas overexpressing mutant epidermal growth factor receptors by systemic administration of monoclonal antibody $(\mathrm{mAb}) 806$, a novel monoclonal antibody directed to the receptor. Cancer Res. 61:5349-5354.

18. Johns, T.G., et al. 2004. Identification of the epitope for the epidermal growth factor receptor-specific monoclonal antibody 806 reveals that it preferentially recognizes an untethered form of the receptor. J. Biol. Chem. 279:30375-30384.

19. Johns, T.G., et al. 2002. Novel monoclonal antibody specific for the de2-7 epidermal growth factor receptor (EGFR) that also recognizes the EGFR expressed in cells containing amplification of the EGFR gene. Int. J. Cancer. 98:398-408.

20. Shigematsu, H., and Gazdar, A.F. 2006. Somatic mutations of epidermal growth factor receptor signaling pathway in lung cancers. Int. J. Cancer. 118:257-262.

21. Panousis, C., et al. 2005. Engineering and characterisation of chimeric monoclonal antibody 806 (ch806) for targeted immunotherapy of tumours expressing de2-7 EGFR or amplified EGFR. Br. J. Cancer. 92:1069-1077.

22. Ji, H., et al. 2006. The impact of human EGFR kinase domain mutations on lung tumorigenesis and in vivo sensitivity to EGFR-targeted therapies. Cancer Cell. 9:485-495.

23. Politi, K., et al. 2006. Lung adenocarcinomas induced in mice by mutant EGF receptors found in human lung cancers respond to a tyrosine kinase inhibitor or to down-regulation of the receptors. Genes Dev. 20:1496-1510.

24. Li, S., et al. 2005. Structural basis for inhibition of the epidermal growth factor receptor by cetuximab. Cancer Cell. 7:301-311.

25. Pedersen, M.W., and Poulsen, H.S. 2006. Mutations in the epidermal growth factor receptor: structure and biological function in human tumors [In Danish]. Ugeskr. Laeger. 168:2354-2361. 
26. Janne, P.A., Engelman, J.A., and Johnson, B.E. 2005. Epidermal growth factor receptor mutations in non-small-cell lung cancer: implications for treatment and tumor biology. J. Clin. Oncol. 23:3227-3234

27. Kobayashi, S., et al. 2005. EGFR mutation and resistance of non-small-cell lung cancer to gefi- tinib. N. Engl. J. Med. 352:786-792.

28. Kobayashi, S., et al. 2005. An alternative inhibitor overcomes resistance caused by a mutation of the epidermal growth factor receptor. Cancer Res. 65:7096-7101.

29. Scott, A.M., et al. 2006. A phase I single dose escalation trial of ch806 in patients with advanced tumors expressing the 806 antigen [abstract]. J. Clin. Oncol. (ASCO Annual Meeting Proceedings). 24(Suppl.):13028.

30. Kubo, S., et al. 2006. Three-dimensional magnetic resonance microscopy of pulmonary solitary tumors in transgenic mice. Magn. Reson. Med. 56:698-703. 